\title{
Dangerous environments
}

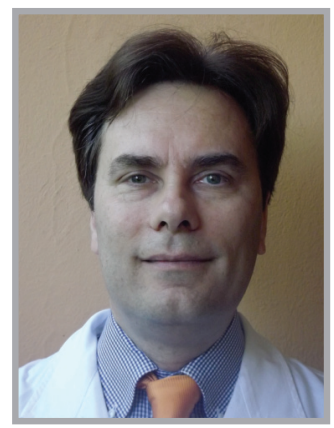

Maurilio Ponzoni* speaks to Roshaine Gunawardana, Managing Commissioning Editor: Dr Maurilio Ponzoni obtained his MD degree and Board of Pathology from the University of Milan. He spent part of his early career in Mayo Clinic, Rochester, MN, USA and New York University Medical Centre, New York, NY, USA. He is the Coordinator of the Hematopathology Diagnostic Area within the Pathology Unit of Ospedale San Raffaele Scientific Institute in Milan, Italy. He is the author of more than 220 papers focused on hematopathology available on PubMed. He is an active member of national and international groups investigating hematologic malignancies.

Your initial degree was in pathology. How did your interest in pathology evolve into a more specific interest in hematopathology \& hematologic malignancies?

I was literally stricken by pathology when I was a MD course student, from the earliest years of medical school. During the same period, I was very attracted by hematology as well. Therefore, I started to study bone marrow biopsies because this takes into account both fields and allowed me to have a close interaction with morphology and its clinical implications. In addition, when the fortuitous possibility arose within my department to also study lymph node biopsies, I saw this opportunity as a sort of natural consequence of my interests. For these reasons, I increasingly dealt with hematopatology during my work and at present this discipline makes up almost my entire routine diagnostic activity. Of course, I have been lucky enough to work within a referral institute for hematologic malignancies, whose patient accrual is large and constantly increasing.

\section{What is the most exciting} development in this field that you have witnessed during your career so far?

This is a difficult question to answer. Actually, I think the most fascinating discovery/development is likely the forthcoming one! Looking back at what I had the privilege to assist with, an important step could be represented by the study reported by our group, which focused on the link between Chlamydophila psittaci infection and the occurrence of marginal zone B-cell lymphoma of orbital adnexa. After the paradigmatic causative link between Helicobacter pylori infection and extranodal marginal zone lymphoma of mucosa associated lymphoid tissue (MALT)type of the stomach, the relationship we described seems the best characterized one with different approaches (please see below) in the context of the relationship 
between bacteria and development of lymphomas. Importantly, these results allowed us to treat these tumors with antibiotics alone, reaching a significant amount of clinical response in these patients.

Another recent amazing finding was the direct visualization of islets of Langerhans within the bone marrow biopsies of diabetic patients undergoing autologous bone marrow pancreatic islet transplantation; this approach is very promising, in particular in comparison with the traditional islet transplantation within the liver for diabetic patients who do not have further chances to produce insulin.

\section{Can you identify any colleagues \& mentors you have had who have influenced your profession \& research directions?}

In addition to my family, I believe that I have to thank the entire Pathology Department in San Raffaele Hospital in Milan for allowing me to work on both diagnostic and research counterparts. Indeed, I must be very grateful to many distinguished scientists that I have had the chance to work with. To give some examples, I would mention Drs Agostino Faravelli, Giorgio Inghirami, Fabio Facchetti and Claudio Doglioni among the pathologists as well as Andrés Ferreri and Riccardo Dolcetti among the clinicians.

You published some works focused on the microenvironment of primary CNS lymphomas. Can you please briefly explain the conclusions of this study? Primary CNS lymphomas (PCNSL) are rare and aggressive neoplasms whose biological features are largely unknown. Recent data are witnessing an important role played by the intratumoral, non-neoplastic microenvironment. Many cell types are responsible of the composition of the microenvironment. In particular, $\mathrm{T}$ lymphocytes seem to cooperate in recruiting neoplastic B-lymphocytes around vessels, which is a common growth pattern in PCNSL. This interaction seems to be mediated by some molecules such as CXCL9 and CXCL12. In addition, $T$ cells may grow themselves around vessels in PCNSL, forming the so-called reactive perivascular T-cell infiltrate. $\mathrm{T}$ cells may form multilayers around vessels: the occurrence of this infiltrate has been associated with a more favorable prognosis in PCNSL.
You have done some work into viral infections/infectious agents \& lymphoma. What, briefly, were your findings \& what further work will need to be done in this area?

The group, to which I belong, investigated the relationship between some bacterial infections and the development of lymphoproliferative disorders. In particular, we deeply characterized the above mentioned link between $C$. psittaci infection and the development of lymphomas of ocular adnexa under several aspects including immunohistochemical, immunofluorescence, electron microscopy and molecular (also laser-capture assisted) approaches, further confirmed by in vivo studies. Equally important, these findings led us to treat these patients with antibiotics alone (i.e., without chemotherapy or radiotherapy) obtaining, in a prospective randomized study, a clinical response in approximately $60 \%$ of them and a clinical complete remission was observed in a substantial fraction of subjects. The active work on this topic is represented by an ongoing prospective study in which the rate of protracted antibiotic therapy in MALT lymphomas of ocular adnexa and the opportunities of retreatment in those cases with infection reoccurrence will be analyzed.

In addition to these findings, we also contributed to the further investigation of the potential link between cutaneous B-cell lymphomas (i.e., not only including MALT lymphomas) and infection with Borrelia burdgoferi; according to our figures, this link does seem to exist only in endemic areas for this microorganism and deserves to be further characterized with additional approaches other than molecular evidences. Finally, we evaluated, for the first time in western countries, the possibility to also treat aggressive lymphomas with antibiotics alone, such as $H$. pylori-related diffuse large B-cell lymphomas of the stomach. In a group of 16 patients, obtaining an overall response rate of $69 \%$, antibiotic therapy did not preclude the use of chemotherapy and radiotherapy in the fraction of relapsing patients, which obtained complete remission as well. Obviously, prospective studies to confirm these findings are needed.

Can you tell our readers a little about the research you are currently focusing on?

Our present research efforts are directed toward a better characterization of microenvironment in hematological neoplasms; in particular, we are 
investigating whether and to what amount nontumoral components may contribute to sustenance, growth and survival of neoplastic elements in lymphoproliferative and myeloproliferative/myelodysplastic disorders. This is a difficult task because it is difficult to ascertain whether a particular cell type could act as a 'driver' instead of a 'passenger' and it is possible that the same individual cell populations may play different roles according to the type of disease considered.

\section{What would you like to achieve in your career in the next few years?}

I think it is important to pursue research on a major individual project in a group where different specialists(s) appertain and may contribute with their own individual skill and experience. It is possible that when performing such a task, some particular aspects of the project will be led by several coprincipal investigators and therefore first/last/corresponding authors may rotate; I see this latter aspect a very important one, because everybody in the group will feel like a central part of the project. A modern and successful research group should arise within the model of disease units (i.e., lymphoma unit, leukemia unit) and include either clinicians (obviously experienced in clinical research), biologists, basic scientists and pathologists, which often are a sort of bridge between the former two professional figures and helpful to coordinate the interdisciplinary connections. Keeping this in mind, the obtained results will have a high chance of being rigorous from a scientific standpoint and with practical implications, because of their intrinsic translational characteristics.

\section{How would you like to see this field progress in the next 5-10 years?}

The technological progress that we have assisted with over the last 20 years, particularly in the last decade, is providing us with a mass of scientific data, which presently are very difficult to analyze in detail and draw a comprehensive rank of their hierarchical degree of importance. On the other hand, some disciplines, in view of these achievements, may be superficially regarded as obsolete. In fact, some people might think that the relationship between new technologies and more traditional disciplines could be a sort of progression with points of no return. Actually, I think that this relationship is indeed mutual, because the brand new data obtained with sophisticated approaches may be able to enhance the traditional parameters used in well-established clinical criteria. To give an easy example, it is clear that, looking at the same slide on the microscope with an interval of 10-20 years, may reveal features unknown in the past that could be easily recognized now even by morphology alone; even more importantly, the possibility to apply immunohistochemical characterization to tissues may amplify and validate many molecules discovered and highlighted by modern approaches such as next-generation sequencing or RNAseq. This is perhaps the reason that explains why pathologists are currently often challenged by basic scientists other than clinicians to join (and to propose themselves) ambitious research programs. Along this line, I think pathology in general, and hematopathology in particular, could offer still uncovered research avenues to people involved in the fields. It is the responsibility of pathologists to understand and take advantage of this exciting opportunity.

\section{Disclaimer}

The opinions expressed in this interview are those of the interviewee and do not necessarily reflect the views of Future Medicine Ltd.

Financial \& competing interests disclosure

$M$ Ponzoni has no relevant affiliations or financial involvement with any organization or entity with a financial interest in or financial conflict with the subject matter or materials discussed in the manuscript. This includes employment, consultancies, honoraria, stock ownership or options, expert testimony, grants or patents received or pending, or royalties.

No writing assistance was utilized in the production of this manuscript. 